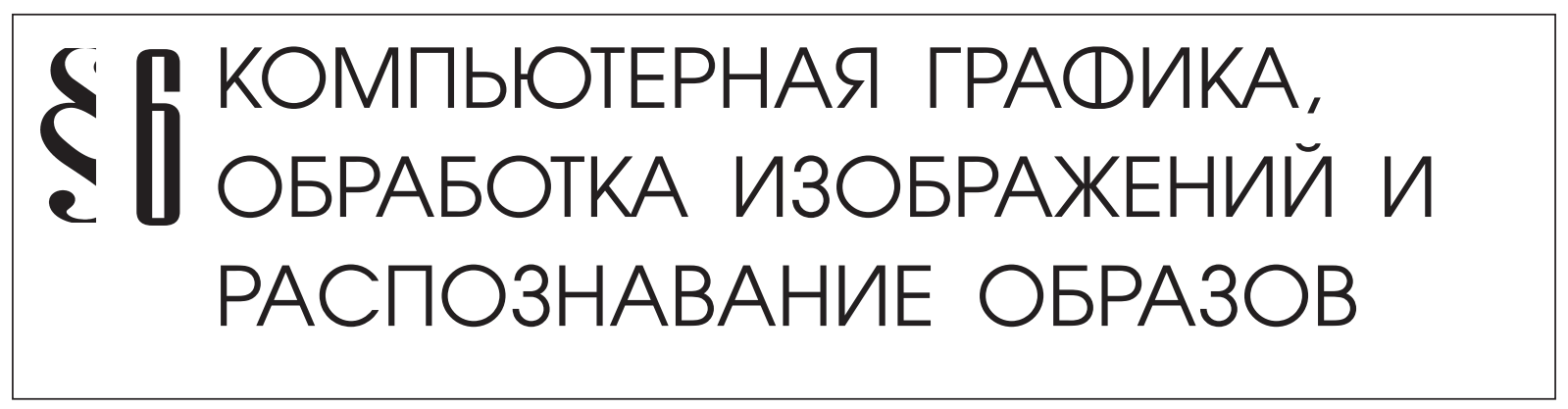

Кревецкий А.В.

\title{
ОСОБЕННОСТИ ФОРМИРОВАНИЯ АССОЦИИРОВАННОГО СПЛОШНОГО ОБРАЗА В ЗАДАЧАХ РАСПОЗНАВАНИЯ ГРУППОВЫХ ТОЧЕЧНЫХ ОБЪЕКТОВ
}

Аннотация: Групповые точечные объекты (ГТО) представляют собой класс изображений, отличающийся несвязностью и вырожденностью своих элементов. Для обнаружения, пространственного разрешения (сегментации, локализации), распознавания и оценки параметров таких объектов в наблюдаемых сценах выполняется связывание элементов ГТО ассочиированным сплошным образом (АСО). Базовой прочедурой формирования ассоциированного образа служит расфокусировка точечной сцены. Для достижения высокой эффективности решения указанных задач по данным зашумленных изображений необходимо обеспечить согласование параметров моделей АСО со свойствами ГТО и параметрами алгоритмов принятия решений. Решение данной задачи достигается методами функционального анализа для поиска экстремумов, теории обработки непрерывных и иифровых изображений для синтеза алгоритмов, дискретной и вычислительной математики для конкретизации численного метода формирования АСО. Для колоколообразной и прямоугольной импульсных характеристик дефокусирующего фильтра получены правила выбора уровня ограничивающего АСО контура с максимальной устойчивостью к ошибкам квантования яркости изображения. Конкретизирована методика согласования параметров модели АСО с плотностью элементов ГТО. Для наглядности и упрощения интерфейса оператора работа с моделями АСО выделен параметр - «радиус» импульсной характеристики фильтра. Получены аналитические связи «радиуса» с порогом локализачии пространственно-компактных ГТО. Синтезирован численный метод базовой прочедуры формирования АСО, отличающийся на один - два порядка больщей производительностью по сравнению с подходом на основе быстрого преобразования Фурье для пространственно-компактных ГТО. Метод базируется на фильтрующих свойствах дельтовидного распределения яркости элементов ГТО и ограничения размеров окна низкочастотного фильтра с учетом числа уровней квантования его импульсной характеристики. Регулярность данной операции для ненулевых отметок наблюдаемой точечной сцены теоретически удобна для распараллеливания вычислений. 
Ключевые слова: групповой точечный объект, ассочиированный сплошной образ, точечные поля, распознавание изображений, анализ точечных сиен, пространственнаялокализация, оптимизация модели, пространственная компактность, быстрые алгоритмы фильтрации, численные методы

Abstract: The issues arising in the implementation of the technical approach to the recognition of the group point object (GPO) images on the basis of the binding elements of the continuous associated images (CAI) are considered. The basic CAl formation models of the point scene defocusing are analyzed. For bell-shaped and rectangular impulse response obtained defocusing filter selection rules limiting circuit CAl level with maximum fault tolerance quantization of the image brightness. Concretize methodology harmonization CAI model parameters with the density of the GPO members. For clarity and simplify the operator interface work with models CAl highlight - radius of the filter impulse response the ratio of the radius with a threshold localize spatially compact objects is obtained. Synthesized numerical method for the formation of base procedure ASO, characterized by one - two orders of magnitude higher performance than with an approach based on the fast Fourier transform for compact GPO. The method is based on the filtering properties of the deltoid brightness distribution GPO elements and limitations of the low pass filter window size based on the number of quantization levels of its impulse response. The results are aimed at ensuring the quality of the detection procedures, permits and GPO recognition in noisy images, set in a one-dimensional, two-dimensional and three-dimensional spaces in their technical implementation.

Keywords: spatial compactness, model optimization, spatial localization, point scene analysis, image recognition, point field, continuous associated images, group point object, fast filtering, numerical methods

\section{Введение}

Специфическим классом образов в области распознавания изображений и анализа сцен являются групповые точечные объекты (ГТО) [1]. ГТО часто ассоциируют с локационными изображениями групп обнаруженных малоразмерных объектов [2], созвездиями [3], характерными точками изображений крупноразмерных объектов [4], представлениями кластеров сигналов в признаковом пространстве [5]. Их изображения представляют собой множество изолированных контрастных по отношению к фону отметок в непрерывном или дискретном пространстве заданной размерности (рис. 1).

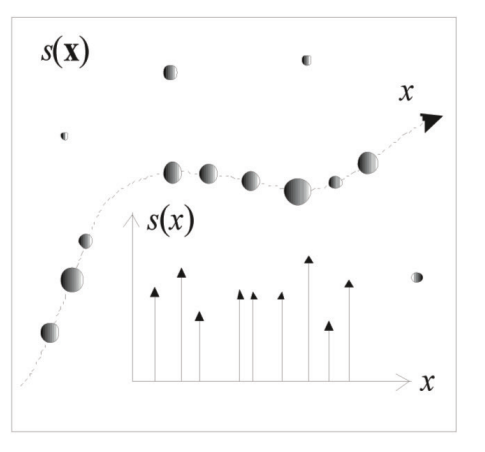

$a$

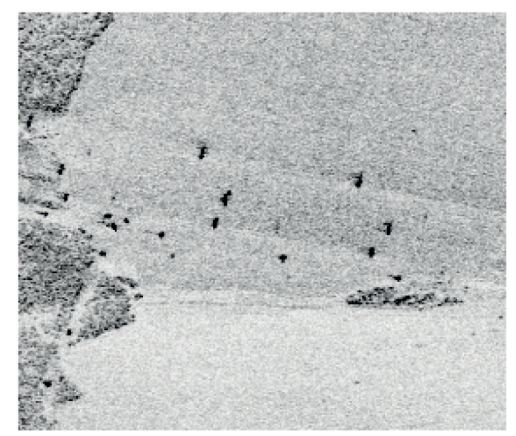

6

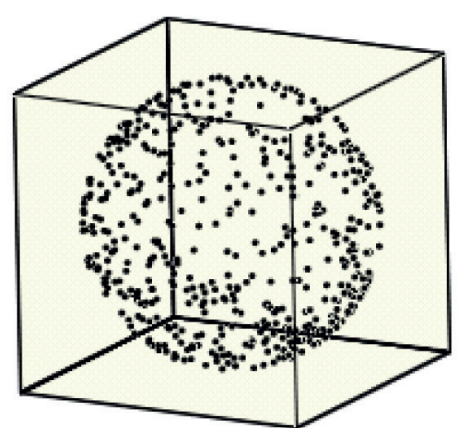

B

$a$ - в криволинейной системе координат; б - на плоскости локационного изображения; в - в трехмерном пространстве 
Форма изображений малоразмерных объектов, из-за соизмеримости с элементом разрешения системы технического зрения, обычно мало информативна и ею пренебрегают, заменяя точкой с координатами в «центре тяжести» [6]. В результате предъявляемая к распознаванию ГТО сцена представляет собой множество

$$
V=\left\{v_{n}\right\}=\left\{\mathbf{x}_{n}, s_{n}\right\}, n=1,2, \ldots, N,
$$

из $N$ точечных объектов с координатами $\boldsymbol{X}_{n}$ и яркостями $S_{n}$. Пространственное распределение яркости сцены будет иметь вид

$$
s(\mathbf{x})=\sum_{n=1}^{N} s_{n} \delta\left(\mathbf{x}-\mathbf{x}_{n}\right), \quad \mathbf{x} \in \mathbf{X},
$$

где $\boldsymbol{X}$ - множество допустимых значений координат в точечной сцене, $\delta(\bullet)$ - символ Кронекера или дельта-функция в зависимости от дискретного или непрерывного пространства.

В зависимости от размерности пространства, в котором задана точечная сцена, могут быть использованы следующие формы представления координат:

1) для одномерного пространства:

$$
\mathbf{x}=x, \quad x \in[0 ; X[\subset \mathbb{R},
$$

где $\boldsymbol{x}$ - действительное число, $\boldsymbol{X}$ - размер интервала наблюдения;

2) для двумерного пространства - точкой на комплексной плоскости $\mathbb{C}$ :

$$
\begin{array}{lll}
\mathbf{x}=x+i y, & x=\operatorname{Re} \mathbf{x}, & y=\operatorname{Im} \mathbf{x}, \\
i=\sqrt{-1}, & x \in[0 ; X[\subset \mathbb{R}, & y \in[0 ; Y[\subset \mathbb{R},
\end{array}
$$

где наблюдаемое изображение - это кадр с размерами $E=X \times Y$ на комплексной плоскости;

3) для трехмерного изображения - векторным кватернионом четырехмерного пространства $\mathbb{H}[9]$ :

$$
\mathbf{x}=0+\mathbf{i} x+\mathbf{j} y+\mathbf{k} z \quad x \in[0 ; X[\subset \mathbb{R} \quad y \in[0 ; Y[\subset \mathbb{R} \quad z \in[0 ; Z[\subset \mathbb{R},
$$

где і, j, k - мнимые единицы (орты) кватернионного пространства, $X \times Y \times Z=E$ - размеры наблюдаемого объема [7].

Для дискретных пространств $x, y, z \in \mathbb{Z}$, т.е. являются целыми числами - номерами элементов разрешения области наблюдения (отсчетов, пикселей и вокселей соответственно).

Сложность распознавания ГТО, как для человеческого сознания, так и для технической системы, во многом определяется несвязностью элементов изображения ГТО [8]. Несвязность элементов затрудняет восприятие изображения ГТО как целостного объекта, обладающего формой. Перспективный подход к распознаванию ГТО основан на методе связывания элементов ГТО ассоциированным сплошным образом [6]. Обобщенные базовые математические модели ассоциированного сплошного образа приводится в работах [1, 6-9]. Однако, вопросы согласования параметров модели со свойствами ГТО 
и прикладными аспектами решаемой задачи, а также вопросы технической реализации данных моделей освещены недостаточно.

В работе предлагается способ конкретизации параметров модели АСО и эффективный в вычислительном плане численный метод формирования АСО.

\section{Модели формирования ассоциированного сплошного образа}

Базовой моделью АСО служит результат пороговой обработки расфокусированного изображения ГТО (рис. 2)

$$
G(\mathbf{x})= \begin{cases}F(\mathbf{x}), & \text { если } F(\mathbf{x}) \geq \beta, \\ 0, & \text { если } F(\mathbf{x})<\beta,\end{cases}
$$

или

$$
\begin{aligned}
& h(\mathbf{x})= \begin{cases}1, & \text { если } F(\mathbf{x}) \geq \beta, \\
0, & \text { если } F(\mathbf{x})<\beta,\end{cases} \\
& F(\mathbf{x})=\int_{X} s(\mathbf{v}) \cdot h_{\phi}(\mathbf{x}-\mathbf{v}) d \mathbf{v},
\end{aligned}
$$

где $h_{\phi}(\mathbf{x})$ - импульсная характеристика низкочастотного дефокусирующего фильтра.

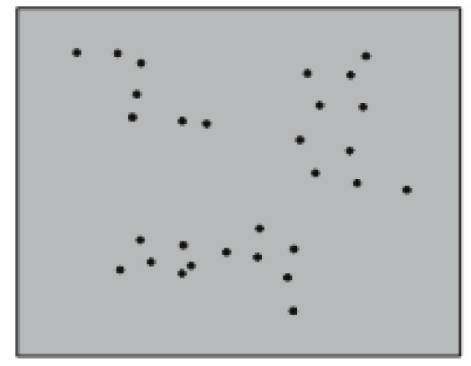

$a$

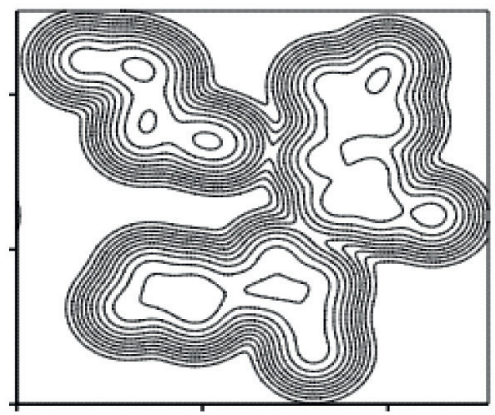

8

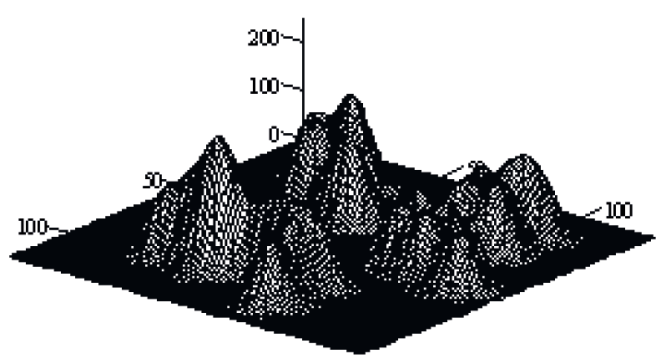

б

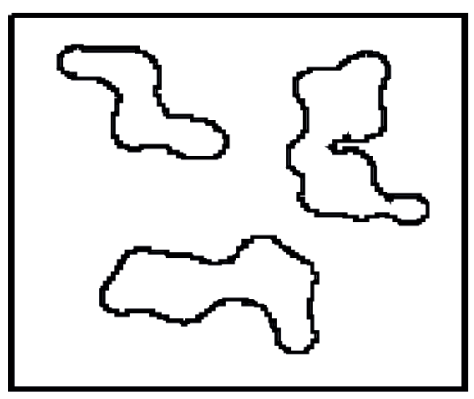

2

Рис. 2. Формирование АСО: $a$ - точечная сцена, $\sigma$ - яркостный рельеф расфокусированного изображения, в - линии уровня рельефа, г - контуры АСО 
Для обеспечения связности АСО и независимости его формы от угловой ориентации объекта на вид импульсной характеристики фильтра накладываются ограничения - монотонность, не возрастание, абсолютная интегрируемость, симметрия (центральная - для одномерного пространства, круговая - для двумерного, сферическая - для 3D).

Примерами таких импульсных характеристик могут служить колоколообразная

$$
h_{\phi}\left(\mathbf{x} \mid r_{0}\right)=\exp \left\{-\alpha|\mathbf{x}|^{2}\right\}
$$

и цилиндрическая

$$
h_{\phi}\left(\mathbf{x} \mid r_{0}\right)=\left\{\begin{array}{l}
1, \text { if }|\mathbf{x}|^{2} \leq \sigma, \\
0, \text { if }|\mathbf{x}|^{2}>\sigma,
\end{array}\right.
$$

где $a$ - декремент затухания, $\sigma$ - радиус ненулевых отсчетов импульсной характеристики.

Приведенная модель АСО задает отображение, которое позволяет представлять модель ГТО моделью АСО в задачах выделения признаков формы ГТО и распознавания формы ГТО. Для пространственно-компактных однородных по плотности ГТО, когда основная информация о форме сосредоточена на границе объекта и фона, достаточным описанием ACO служит его контур [6]. Кроме того, контур локализует область расположения ГТО и связывает своей внутренней областью множество точек в один ГТО.

\section{Выбор порогового уровня для построения контура АCO}

Контуры на рис. 1,2 соответствуют конкретной линии яркостного уровня рельефа рис. 1,6 из множества линий уровня. Вопрос выбора уровня $\beta$ требует обоснования. Низкий уровень приедет к сильной сглаженности контуров АСО и «прилипанию» точечных отметок, не принадлежащих ГТО (например, ложных) и даже слиянию соседних ГТО. Высокий уровень приведет к распаду ГТО на части (см. рис. 2,8).

Из практики распознавания контуров крупноразмерных объектов [6] известно, что минимальная ошибка сегментации однородных по яркости объектов достигается на уровне максимального градиента яркости.

Положим, что радиус импульсной характеристики дефокусирующего фильтра согласован с плотность точек ГТО, а яркость отдельных точечных объектов не информативна, т.е. $s_{n}=1, \forall n=1 \ldots N$. Тогда склон внешней границы АСО будет, в основном, определятся вкладом ближайшей точки ГТО при использовании (9) и (10). В этом случае с достаточной для технической реализации точностью уровень максимального градиента внешнего склона можно найти из уравнения

$$
\frac{d^{2} h(x)}{d x^{2}}=4 \alpha^{2} x^{2} \exp \left(-\alpha x^{2}\right)-2 \alpha \exp \left(-\alpha x^{2}\right)=0,
$$

т.е. приравняв вторую производную колоколообразной импульсной характеристики (9) к нулю. Решением уравнения будет значение аргумента 


$$
x_{g}=\underset{x}{\arg \max } \frac{d h(x)}{d x}=\frac{1}{\sqrt{2 \alpha}} .
$$

Подставив найденное решение уравнения (11) в выражение для импульсной характеристики (9), получим искомое значение яркостного уровня для выделения контура ACO:

$$
\beta=h\left(x_{g}\right)=\exp (-1 / 2) \approx 0,607 .
$$

Для фиксированной яркости точечных отметок $s_{n}=s_{\mathrm{dot}}, n=1,2, \ldots, N$ значение нормированного порога $\beta$ увеличивается прямо пропорционально $S_{\mathrm{dot}}$. Такая ситуация характерна при решении задачи пространственной локализации ГТО [8], оценки занимаемой им площади, структурного анализа ГТО. По опыту автора, при использовании видеопамяти с глубиной 8 бит на пиксел, чтобы переполнения разрядности при формировании ACO не были частыми и не нарушалась реалистичность индикации ACO, целесообразно назначать $S_{\text {dot }}$ в диапазоне 63...127. Очевидно, что для импульсной характеристики (10) выбор порога $\beta$ гораздо проще: $0<\beta<1$.Этот вид фильтра приводит купрощению меры компактности точек [8].

\section{Согласование декремента затухания фильтра с плотностью точек ГТО}

Для оперирования более привычными для более широкого круга пользователей размерностями при описании степени расфокусировки точечной сцены при формировании ACO выразим декремент затухания а через параметр б, имеющий размерность расстояния: $\alpha=1 /(2 \sigma$. Это важно, например, для организации интуитивно понятного интерфейса оператора системы технического зрения [11]. Для краткости назовем $\sigma$, так же как в (10), «радиусом» импульсной характеристики. Тогда $x_{g}=\sigma$. Чтобы ассоциированный сплошной образ связывал два точечных объекта, расположенных друг от друга на расстоянии $r \leq r_{0}$, необходимо, чтобы в центре между ними отклик фильтра превышал порог $\beta$ (см. рис. 3).

Сплошной линией на рис. 3 показан отклик фильтра для одиночного точечного объекта, пунктиром - распределение яркости расфокусированного изображения двух точечных объектов на удалении $r=r_{0}=2$. Горизонтальной линией отмечен найденный ранее пороговый уровень $\beta$ выделения контура АСО. Случай на рис. 3 иллюстрирует минимальный радиус импульсной характеристики фильтра, при котором происходит связывание двух точечных объектов одним АСО для указанного расстояния.

В общем виде для произвольного расстояния $r_{0}$ радиус $\sigma$ назначается из уравнения

$$
2 \exp \left(-\frac{\left(r_{0} / 2\right)^{2}}{2 \sigma^{2}}\right)=\beta=\exp \left(-\frac{1}{2}\right) .
$$

где $r_{0}$ - пороговое расстояние пространственной локализации (группировки) точек. Подставив $2=\exp (\ln 2)$, после логарифмирования получим эквивалентное уравнение 


$$
\frac{r_{0}^{2}}{8 \sigma^{2}}-\ln 2=\frac{1}{2}
$$

После приведения к общему знаменателю, $r_{0}^{2}=\sigma^{2} 4(1+2 \ln 2)$, получим искомое минимальное значение радиуса импульсной характеристики фильтра

$$
\sigma=\frac{r_{0}}{2 \sqrt{1+\ln 4}} \approx \frac{r_{0}}{3,09} .
$$

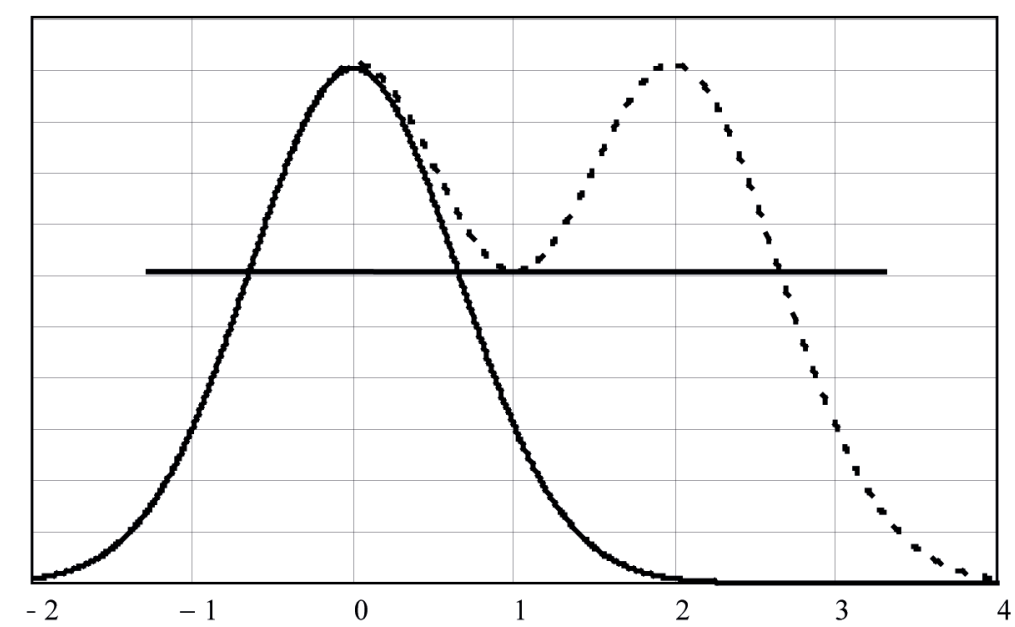

Рис. 3. К правилу выбора радиуса импульсной характеристики фильтра при формировании АСО

В изображениях реальных ГТО расстояния между ближайшими точками - случайная величина. Чтобы учесть разброс таких расстояний при решении задачи пространственной локализации ГТО, величину радиуса б следует назначать с коэффициентом запаса $k_{1}$ относительно параметров закона распределения случайной величины: $r_{0}=k_{1} r$. В роли $r$ может выступать, например, математическое ожидание $\bar{r}$, медиана $r_{\text {med }}$, мода $r_{\text {mod }}$ или их выборочные оценки.

\section{Численный метод реализации модели АCO}

Анализ операций формирования АСО показывает, что самая трудоемкую часть процедуры составляет расфокусировка, т.е. низкочастотная фильтрация точечной сцены (8). Как известно, быстрые алгоритмы линейной фильтрации цифровых изображений строятся на основе произведения спектров наблюдаемого изображения и апмплитудно-фазочастотной характеристики фильтра, получаемых на основе ортогональных разложений сигнала и импульсной характеристики [10] . Самым доступным, благодаря встроенности в популярных средах моделирования, из эффективных методов этого подхода можно считать быстрое преобразование Фурье. Трудоемкость $T$ этого метода с поправкой $k_{\mathrm{a}}$ на 
неучтенные операции связана с размерностью сигнала $n$ соотношением $T=k_{\mathrm{a}} 2 n \log n$. Прямое вычисление свертки (8) в общем случае пропорционально квадрату размерности сигнала, т.е. проигрывает.

Подставим описание точечной сцены (2) в выражение для свертки (8). В результате фильтрующего действия $\delta$-функции получим

$$
F(\mathbf{x})=\int_{X} s(\mathbf{v}) \cdot h_{\mathrm{o}}(\mathbf{x}-\mathbf{v}) d \mathbf{v}=\sum_{n=1}^{N} s_{n} \cdot h_{\mathrm{o}}\left(\mathbf{x}-\mathbf{x}_{n}\right),
$$

а при неинформативной яркости точеных отметок ГТО

$$
F(\mathbf{x})=\sum_{n=1}^{N} h_{\hat{o}}\left(\mathbf{x}-\mathbf{x}_{n}\right) .
$$

Таким образом, отклик фильтра на точечную сцену представляет собой сумму $N$ смещенных в координаты точечных объектов копий импульсных характеристик фильтра. Т.е. для (17) операции умножения не требуются вовсе. Трудоемкость процедуры формирования ACO с такой организацией процесса фильтрации с поправкой $k_{\mathrm{b}}$ на неучтенные операции будет пропорциональна числу точечных отметок в наблюдаемой сцене $N$ и размеру окна фильтра $m$ :

$$
T_{\mathrm{b}}=k_{\mathrm{b}} m N \text {. }
$$

Размер окна фильтра (9) или (10) для пространственно-компактных ГТО можно существенно сократить по сравнению с размерами точечной сцены, т.к. расстояния между соседними элементами ГТО существенно меньше размеров наблюдаемого изображения. Для определения размера окна достаточно определить, на каком расстоянии $r_{M}$ значение импульсной характеристики (9) станет равной половине младшего уровня ее квантования $\triangle$ :

$$
r_{M}=\underset{x}{\arg }\left[\exp \left(-\frac{|x|^{2}}{2 \sigma^{2}}\right)=\frac{\Delta}{2}\right] .
$$

Решением уравнения будет значение

$$
r_{M}=\sigma \sqrt{-2 \ln (\Delta / 2)}
$$

Размер окна цифрового фильтра для симметрии импульсной характеристики необходимо выбирать нечетным. Тогда он составит $2 M+1$, где $M-$ ближайшее целое к $r_{M}$. Для удобства программной реализации окно фильтра принимают квадратным. В этом случае фильтрация точечной сцене сводится к циклической (по числу точечных отметок $N$ ) операции накопления яркости импульсной характеристики фильтра в предварительно обнуленной видеопамяти: 


$$
\begin{gathered}
F_{m}\left(x_{n}+m\right)=F_{m}\left(x_{n}+m\right)+h(m), m=-M \ldots M, \\
F_{i j}\left(x_{n}+i, y_{n}+j\right)=F_{i j}\left(x_{n}+i, y_{n}+j\right)+h(i, j), i, j=-M \ldots M, \\
F_{i j}\left(x_{n}+i, y_{n}+j, \mathrm{z}_{n}+k\right)=F_{i j}\left(x_{n}+i, y_{n}+j, \mathrm{z}_{n}+k\right)+h(i, j, k), i, j, k=-M \ldots M,
\end{gathered}
$$

соответственно для одномерного, двухмерного и трехмерного пространства, в котором задана точечная сцена.

Приведем оценку трудоемкости данного численного метода при конкретных значениях рассмотренных параметров. Так, при пороге пространственной локализации $r_{0}=32$ пикселя и шаге квантования импульсной характеристики $\Delta=1 / 32$, ширина окна фильтра составит 61 пиксель. Для двумерной точечной сцены любых размеров с числом точечных объектов $N=50$ трудоемкость процедуры дефокусировки составит $T_{\mathrm{b}} \approx k_{\mathrm{b}} \cdot 1,8 \cdot 10^{5}$ операций. Для сравнения, фильтрация на основе БПФ при размерах кадра двумерного изображения $X \times Y=512 \times 512$ составит $T=k_{\text {a }} \cdot 2,8 \cdot 10^{6}$ операций, т.е. приблизительно на порядок больше. Для изображения $X \times Y=1024 \times 1024-T=k_{\mathrm{a}} \cdot 1,3 \cdot 10^{7}$ и проигрыш будет уже около двух порядков.

\section{Заключение}

Групповые точечные объекты представляют собой класс изображений, отличающийся несвязностью и вырожденностью своих элементов. Для обнаружения, пространственного разрешения (сегментации, локализации), распознавания и оценки параметров таких объектов в наблюдаемых сценах выполняется связывание элементов ГТО ассоциированным сплошным образом (АСО). Базовой процедурой формирования ассоциированного образа служит расфокусировка точечной сцены. Для

Оптимальным пороговым уровнем контура в модели АСО с точки зрения устойчивости формы ассоциированного образа к ошибкам квантования по яркости и минимума ошибок пространственной локализации ГТО является уровень максимального градиента. Для колоколообразной и прямоугольной импульсных характеристик дефокусирующего фильтра получены упрощенные, но значимые для практики, правила выбора этого уровня.

Качество обнаружения, разрешения и распознавания ГТО во многом определяются согласованностью параметров модели АСО с плотностью точечной сцены. Для обеспечения интуитивной понятности интерфейса оператора при работе с типовыми фильтрами модели АСО введен параметр - «радиус» импульсной характеристики фильтра, - для которого соотношения с параметрами плотности ГТО выводятся аналитически. В качестве исходных данных могут использоваться среднее значение, мода или медиана распределения ребер минимального остовного дерева ГТО.

Особенность изображений ГТО, заключающееся в дельтовидном распределении яркости их элементов, позволяет представить процесс формирования АСО в виде суммы небольшого (по числу точечных отметок в сцене) числа смещенных в координаты точеч- 
ных объектов копий импульсной характеристики фильтра. Для пространственно-компактных групп, когда расстояние между соседними точечными объектами существенно меньше размеров сцены, пропорционально сокращается и окно фильтра - область с ненулевыми отсчетами импульсной характеристики. Размер окна фильтра в явном виде определяется по выше названному радиусу импульсной характеристики и числу уровней ее квантования. Все это позволяет синтезировать численный метод базовой процедуры формирования ACO, обладающий на один - два порядка большей производительностью, по сравнению с процедурами на основе быстрого преобразования Фурье, и очень простой базовой операцией.

Регулярность данной операции для ненулевых отметок наблюдаемой точечной сцены теоретически удобна для распараллеливания вычислений. В дальнейшем планируется получить параллельную реализацию полученного численного метода для многопроцессорной системы.

\section{Работа выполнена при финансовой поддержке РФФИ, проект № 16-01-00451.}

\section{Библиография :}

1. Точечные поля и групповые объекты / Я. А Фурман, А. А Роженцов, Р. Г. Хафизов, Д. Г. Хафизов, А. В. Кревецкий, Р. В. Ерусланов; под общ. ред. Я. А. Фурмана. - М: ФИЗМАТЛИТ, 2014. - 440 с.

2. Верба В.С. Обнаружение наземных объектов. Радиолокационные системы обнаружения и наведения воздушного базирования. - М.: Радиотехника, 2007. - 360 с.

3. Анисимов В.В., Курганов В.Д., Злобин В.К. Распознавание и цифровая обработка изображений. - М.: Высш. шк., 1983. -295 C.

4. Цифровая обработка видеоизображений / А.А. Лукъяница, А.Г.Шишкин. - М.: «Ай-Эс-Эс Пресс», 2009. -518 c.

5. Мандель И. Д. Кластерный анализ. - М.: Финансы и статистика, 1988. - 176 с.

6. Введение в контурный анализ; приложения к обработке изображений и сигналов / Я.А. Фурман, А.В. Кревецкий, А.К. Передреев, и др.; под ред. Я.А. Фурмана. - 2-е изд. - М.: ФИЗМАТЛИТ, 2003. - 592 с.

7. Комплекснозначные и гиперкомплексные системы в задачах обработки многомерных сигналов / Я.А. Фурман, А.В. Кревецкий, А.А. Роженцов, и др.; под ред. Я.А. Фурмана. - М.: ФИЗМАТЛИТ, 2004. - 456 с.

8. Кревецкий А.В. Инвариантные к форме обнаружение и пространственная локализация групп точечных объектов в трехмерном пространстве // Вестник МарГту. Радио-технические и инфокоммуникационные системы. - Йошкар-0ла: Изд-во МарГТУ, 2011.- №1. - С. 47-53.

9. Кревецкий А.В., Чесноков С.Е. Кодирование и распознавание изображений множеств точечных объектов на основе моделей физических полей// Автометрия, 2002. - №3. - С. 80-89.

10. Блейхут Р. Быстрые алгоритмы цифровой обработки сигналов: Пер. с англ.—М.: Мир, 1989. - 448 c. 
11. Чесноков С.Е., Кревецкий А.В.,Уржумов Д.В.,ИпатовЮ.А.Архитектурасистем комплексногодешифрирования изображений аэрокосмических изображений подстилающей поверхности земли в реальном масштабе времени // Вестник МарГТУ. Радиотехнические и инфокоммуникационные системы. - Йошкар-Ола: Изд-во МарГТУ, 2012. - №1. - C. 47-59.

\section{References:}

1. Tochechnye polya i gruppovye ob"ekty / Ya. A Furman, A. A Rozhentsov, R. G. Khafizov, D. G. Khafizov, A. V. Krevetskii, R. V. Eruslanov; pod obshch. red. Ya. A. Furmana. - M: FIZMATLIT, 2014. - 440 s.

2. Verba V.S. Obnaruzhenie nazemnykh ob"ektov. Radiolokatsionnye sistemy obnaruzheniya i navedeniya vozdushnogo bazirovaniya. - M.: Radiotekhnika, 2007. - 360 s.

3. Anisimov V.V., Kurganov V.D., Zlobin V.K. Raspoznavanie i tsifrovaya obrabotka izobrazhenii. - M.:Vyssh. shk., 1983. $-295 \mathrm{~s}$.

4. Tsifrovaya obrabotka videoizobrazhenii / A.A. Luk"yanitsa, A.G.Shishkin. - M.: «Ai-Es-Es Press», 2009. - 518 s.

5. Mandel' I. D. Klasternyi analiz. - M.: Finansy i statistika, 1988. - $176 \mathrm{~s}$.

6. Vvedenie v konturnyi analiz; prilozheniya k obrabotke izobrazhenii i signalov / Ya.A. Furman, A.V. Krevetskii, A.K. Peredreev, i dr.; pod red. Ya.A. Furmana. - 2-e izd. - M.: FIZMATLIT, 2003. - 592 s.

7. Kompleksnoznachnye i giperkompleksnye sistemy v zadachakh obrabotki mnogomernykh signalov / Ya.A. Furman, A.V. Krevetskii, A.A. Rozhentsov, i dr.; pod red. Ya.A. Furmana. - M.: FIZMATLIT, 2004. - 456 s.

8. Krevetskii A.V. Invariantnye k forme obnaruzhenie i prostranstvennaya lokalizatsiya grupp tochechnykh ob"ektov v trekhmernom prostranstve // Vestnik MarGTU. Radio-tekhnicheskie i infokommunikatsionnye sistemy. - loshkar-0la: Izd-vo MarGTU, 2011.- №1. - S. 47-53.

9. Krevetskii A.V., Chesnokov S.E. Kodirovanie i raspoznavanie izobrazhenii mnozhestv tochechnykh ob"ektov na osnove modelei fizicheskikh polei// Avtometriya, 2002. - №3. - S. 80-89.

10. Bleikhut R. Bystrye algoritmy tsifrovoi obrabotki signalov: Per. s angl.-M.: Mir, 1989. - 448 s.

11. Chesnokov S.E., Krevetskii A.V., Urzhumov D.V., Ipatov Yu.A. Arkhitektura sistem kompleksnogo deshifrirovaniya izobrazhenii aerokosmicheskikh izobrazhenii podstilayushchei poverkhnosti zemli v real'nom masshtabe vremeni // Vestnik MarGTU. Radiotekhnicheskie i infokommunikatsionnye sistemy. - loshkar-0la: Izd-vo MarGTU, 2012. №1. - S. 47-59. 